\title{
A case of paraplegia due to asymptomatic varicella-zoster virus infection in AIDS patient unexpectedly diagnosed by CSF metagenomic next-generation sequencing
}

Zhiman $\mathrm{Xie}^{1 \dagger}$, Jingzhen Lai ${ }^{2,3+}$, Chuanyi Ning ${ }^{2,4 \dagger}$, Guangjing Ruan ${ }^{1}$ and Hao Liang ${ }^{2,3 *}$ (B)

\begin{abstract}
Background: Varicella-zoster virus (VZV) infection may induce central nervous system complications in HIV/AIDS patients. However, it is rare to have paraplegia caused by VZV infection but no herpes zoster clinically. Asymptomatic VZV infection in HIV/AIDS patient increased the difficulty of diagnosis.

Case presentation: We reported a 41-year-old male AIDS patient with rare asymptomatic VZV infection-induced paraplegia after his anti-retroviral therapy initiation. MRI of the spinal cord showed the morphology of the thoracic spinal cord was irregular and locally inflated. The patient was confirmed as VZV induced thoracic myelomyelitis by using the cerebrospinal fluid for metagenomic next-generation sequencing (mNGS).
\end{abstract}

Conclusions: mNGS may contribute to disease diagnosis for asymptomatic VZV infection-induced myelitis.

Keywords: HIV, AIDS, Varicella-Zoster virus, Paraplegia, mNGS

\section{Background}

Varicella-zoster virus (VZV) is also known as Human alphaherpesvirus_3. VZV infection can cause primary varicella infection (chickenpox) in children, meanwhile a few cases will develop to herpes zoster in adults after reactivation [1]. The incidence of herpes zoster in antiretroviral therapy (ART)-naïve human immunodeficiency virus (HIV) infected patients can reach 9.4\% [2]. In HIV/ AIDS patients, the risk of VZV infection increased and induced many related complications, including central nervous system (CNS) complications [3]. However, it is

\footnotetext{
*Correspondence: lianghao@gxmu.edu.cn

†Zhiman Xie, Jingzhen Lai and Chuanyi Ning contributed equally to this work

${ }^{3}$ Guangxi Collaborative Innovation Center for Biomedicine, Life Sciences Institute, Guangxi Medical University, No. 22 Shuangyong Road, 530021 Nanning, Guangxi, China

Full list of author information is available at the end of the article
}

rare to have paraplegia caused by direct invasion of the spinal cord by VZV but no herpes zoster clinically in HIV-VZV coinfected patients. Here we present a rare case of myelitis due to VZV infection without VZVrelated clinical symptoms in AIDS patients who were diagnosed by cerebrospinal fluid (CSF) MAPMI ${ }^{\mathrm{TM}}$, a metagenomic next-generation sequencing technology (mNGS).

\section{Case presentation}

A 41-year-old male was presented in our hospital on February 15th, 2020, for bilateral lower limb paralysis for more than 10 days. In the middle of January 2020, he was admitted in the local hospital due to he had fever for more than one month. He was diagnosed with AIDS, bacterial pneumonia, and cytomegalovirus (CMV) infection in the local hospital. Seven hundreds copies $/ \mathrm{ml}$ of CMV were detected in urine On January 16th, and 
$5.77 \times 10^{3}$ copies $/ \mathrm{ml}$ of $\mathrm{CMV}$ were detected in sputum on January 20th. After antimicrobial and anti-CMV treatment, the patient's condition improved. The combined ART regimen of Nevirapine, Lamivudine, and Tenofovir was taken on January 27th, 2020. In the following days after cART initiation, he experienced weakness of lower limbs and developed paralysis of both legs and incontinence in a short time. He reported no skin lesions, fear of cold, fever, dizziness, headache, vomiting, limb convulsions, abdominal pain, diarrhea, or any other discomfort. He also reported no special medical history or trauma history. We gave him a physical examination when admission: his lower limb muscle tension was low, and the muscle strength was level 0 . But the passive range of motion of all joints of the body was normal. His sensation disappeared in the sacrococcyx, buttocks, front and lateral thighs, back and medial sides, calves, and feet. However, pathological emission did not elicit.

The clinical tests after admission showed that the $\mathrm{CD}^{+} \mathrm{T}$ cell count was 35 cells $/ \mu \mathrm{L}$, white blood cells (WBC) count was $4.8 \times 10^{9} / \mathrm{L}$, red blood cell (RBC) count was $3.24 \times 10^{12} / \mathrm{L}$, hemoglobin $(\mathrm{Hb})$ was $90 \mathrm{~g} / \mathrm{L}$, Platelets (PLT) was $259 \times 10^{9} / \mathrm{L}$, and the percentage of neutrophile granulocyte was $76.4 \%$. No abnormalities were found in liver and renal function tests. Screening for hepatitis B virus infection, hepatitis $C$ virus infection, and syphilis were negative. Neither was the Mycobacterium tuberculosis (Mtb) infecting T cells test. DNA tests for CMV and Mtb in blood and CSF were negative, too. CSF examination showed the opening pressure was $60 \mathrm{~mm} \mathrm{H}_{2} \mathrm{O}$ by lumbar puncture, the Pandy test was weakly positive, $16.00 \times 10^{6} \mathrm{WBC} / \mathrm{L}$, and a normal level of adenosine deaminase (2.01 U/L). The biochemical indicators of CSF revealed protein concentration was $960.60 \mathrm{mg} / \mathrm{L}$ (normal range, 150-450 mg/L), normal level of glucose $(3.59 \mathrm{mmol} / \mathrm{L})$, and chloride concentration was $111.30 \mathrm{mmol} / \mathrm{L}$ (normal range, $120-132 \mathrm{mmol} / \mathrm{L}$ ). No bacteria or fungi was found in CSF smear and culture. MRI of the brain and electrocardiography were normal. However, MRI of the spinal cord showed the morphology of the thoracic spinal cord was irregular and locally inflated, and the signals of T1 and T2 were slightly longer (Fig. 1a-c). It indicated infection.

To figure out the pathogens that induced the infection in the spinal cord, the CSF was used for the super broad-spectrum pathogenic microorganisms mNGS by MAPMITM (CapitalBio MedLab Co. LTD, Beijing, China). The mNGS was done on BioelectronSeq4000
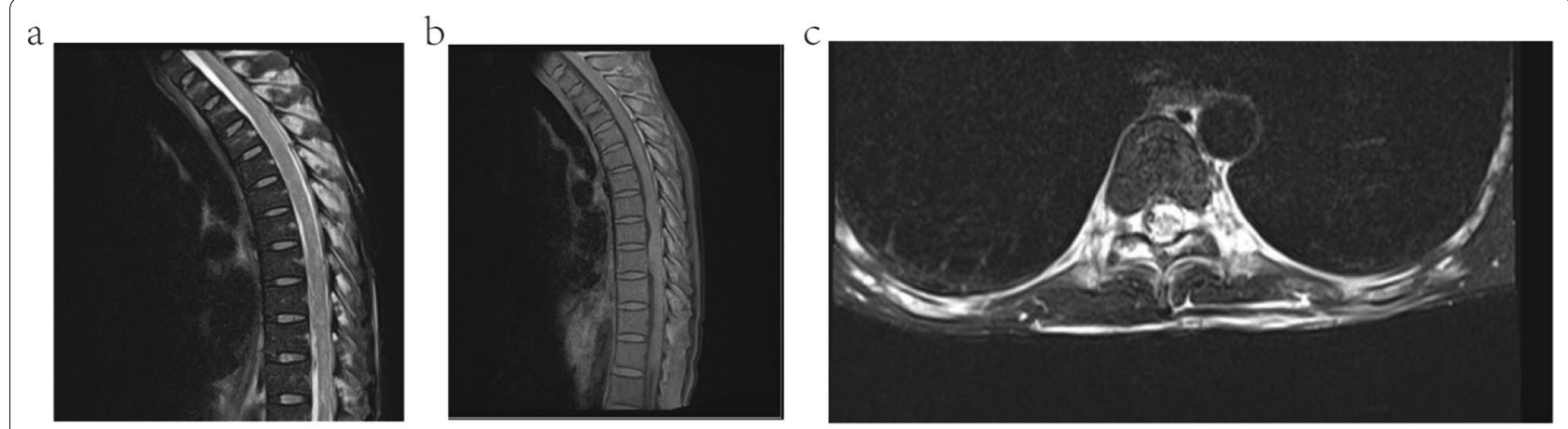

d
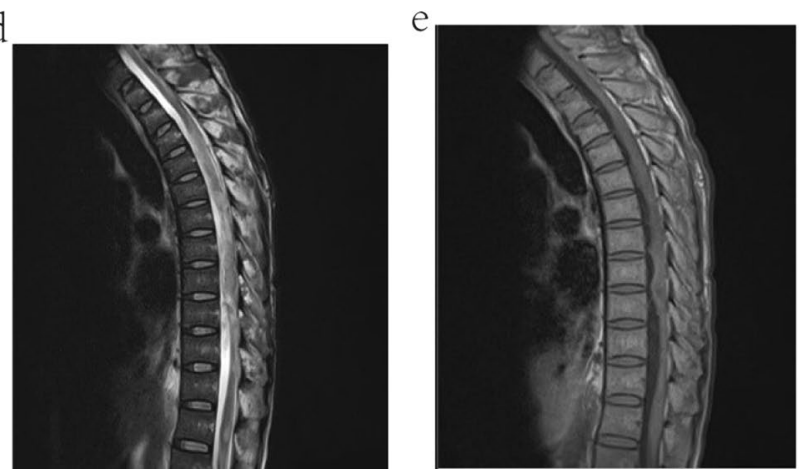

Fig. 1 Spinal cord MRI and the mNGS result of the patient. $\mathbf{a}$ and $\mathbf{d}$ were the spinal cord segmental MRI T2-weighted image of the patient, $\mathbf{b}$ and $\mathbf{e}$ were the spinal cord segmental MRI T1-weighted image of the patient, $\mathbf{c}$ was the axial spinal cord segmental MRI T2-weighted image of the patient. a-c were recorded on February 17th, 2020. a-c showed that the thoracic spinal cord was irregular in shape with local enlargement and spotted and patchy hypersignal, which indicated edema or infection. $\mathbf{d}$, e were recorded on April 3rd, 2020. Compared to $\mathbf{a}, \mathbf{b}$ the edema spinal cord was reduced after treatment 
platform (CapitalBio). After quality filtering, eliminating replicate reads, removing reads matching human genome sequence, reads were classified against the NCBI reference/representative bacterial, archaeal, and fungal genomes, as well as genomes of DNA viruses infecting humans and DNA bacteriophages. Of 25,337,336 quality filtered sequencing reads, 23,485,313 were human, 53,502 were assigned to microbe, and 3935 were mapped on Human_alphaherpesvirus_3 (VZV). The VZV genome coverage was $95.55 \%$, and the estimated VZV DNA concentration was $2.6 \times 10^{3}$ copies $/ \mathrm{ml}$ (computed from VZV to human read counts ratio combined with a TaqMan-based quantification of human DNA). Screening results for bacteria, fungi, and parasites were negative. The results indicated the pathogen was VZV (three representative DNA sequences got from mNGS and the reference sequence were showed in Additional file 1 and Additional file 2). The patient was confirmed as AIDS with VZV induced thoracic myelomyelitis.

After admission, he was treated with sodium phosphonate $3.0 \mathrm{~g} / 3$ times a day for 50 days, adjuvanted with traditional Chinese acupuncture for more than a month to control the symptom. Acyclovir $0.5 \mathrm{~g} / 3$ times a day was used for 22 days after he was diagnosis as VZV infection by mNGS. The MRI reexamination of the spinal on April 3rd showed that the edema of the lesion was reduce (Fig. 1d, e). However, his disease progression did not improve or get worse after more than 1 month's treatment. Therefore, he abandoned further treatment automatically and was discharged from our hospital.

\section{Discussion and conclusions}

VZV is an enveloped DNA virus, which can invade tissues of ectodermal origin, including skin, mucous membranes, and nerve tissue. Typical VZV-related manifestations include rash and pain [4]. VZV infection can cause a wide range of neurological diseases, such as postherpetic neuralgia, facial paralysis with Ramsay-Hunt syndrome, meningitis, encephalitis, myelitis, encephalomyelitis, and cerebrovascular disease, etc. [5-8].

The risk of VZV infection in HIV/AIDS patients was 2.5-fold higher compared with the general population [9]. It was estimated that subclinical reactivation of VZV would be found in $15 \%$ of HIV-positive subjects, most of whom were asymptomatic [10]. The higher incidence of zoster in HIV/AIDS patients was reported to be associated with lower CD4 counts [11]. Additionally, ART initiation was reported to be associated with zoster occurrence in HIV/AIDS patients [12]. VZV meningitis may be accompanied with or without shingles, but VZV myelitis without a rash is rare. It is even rarer that VZV myelitis induced paraplegia without rash. The diagnosis of VZV myelitis is mostly based on the time correlation between typical skin lesions and myelopathy. MRI examination is helpful for diagnosis and differential diagnosis. However, it is difficult to diagnose VZV myelitis clinically without a rash. For VZV myelitis induced paraplegia patient without rash, the lack of an effective direct detection method for virus in CSF may result in poor prognosis or death. It was difficult to isolate the virus in the blood and CSF of patients, so VZV infection could be diagnosed through typical rash, clinical symptoms of spinal cord injury and MRI.

The clinical manifestations of the reported case didn't include chills and fever, dizziness, headache, abdominal pain, diarrhea, or herpes zoster. Paraplegia is rarely the only manifestation of VZV infection. Therefore, the diagnosis cannot be made based on the manifestations. Here, we have made a pioneering attempt to isolate the pathogen DNA in CSF for mNGS. mNGS a highthroughput sequencing technology, has been applicated in clinical diagnosis for some unclear pathogens. With the application of mNGS, we found the sequences of VZV with an estimated viral load of $2.6 \times 10^{3}$ copies/ $\mathrm{mL}$, and no other viruses, bacteria, fungi, or other pathogens were found. The sequencing result confirmed the diagnosis of the patient, backing by his symptoms and clinical tests. We suggest that a routine test for VZV DNA in CSF should be established for the HIV-infected patients with neurological diseases, such as PCR for VZV DNA.

The patient was treated with sodium phosphonate $3.0 \mathrm{~g} / 3$ times a day and acyclovir $0.5 \mathrm{~g} / 3$ times a day during hospitalization. Acyclovir intravenous treatment is a recommended regimen for VZV infection [13]. Early antiviral and steroid therapy are the most important factor in the good prognosis of such patients. But steroid was not given in this case, because its side effects and it may mask other symptoms of unidentified opportunistic infections. In addition, it could be that intravenous acyclovir interferes with the rash, which leads to no rash manifestation in this patient.

Here, we reported a rare case of myelitis paraplegia caused by VZV without herpes-related symptoms, and share examples of metagenomic sequencing used in clinical diagnosis of difficult diseases, to provide a reference for other similar diseases.

\section{Abbreviations}

AIDS: Acquired Immune Deficiency Syndrome; CART: Combined Anti-Retroviral Therapy; CMV: Cytomegalovirus; CNS: Central Nervous System; CSF: Cerebrospinal Fluid; DNA: Deoxyribonucleic Acid; Hb: Hemoglobin; HIV: Human Immunodeficiency Virus; mNGS: metagenomic Next-Generation Sequencing; MRI: Magnetic Resonance Imaging; PLT: Platelets; RBC: Red Blood Cell; VZV: Varicella-Zoster Virus; WBC: White Blood Cells. 


\section{Supplementary Information}

The online version contains supplementary material available at https://doi. org/10.1186/s12879-021-06611-9.

Additional file 1. Three representative DNA sequences got from CSF by mNGS

Additional file 2. The reference sequence for sequencing analysis.

\section{Acknowledgements}

None.

\section{Authors' contributions}

ZX and GR collected and analyzed the data of the patients. JL analyzed the data and wrote the manuscript. CN and $\mathrm{HL}$ conceptualized the report and provided overall supervision. All authors read and approved the final manuscript.

\section{Funding}

This study was funded by the National Natural Science Foundation of China (81760602 and 81803295), Guangxi Natural Science Foundation (2018GXNSFAA138031), and the Research Basic Ability Enhancement Project of Young and Middle-aged Teachers in Guangxi Universities in 2021 (2021 KY0081). The funding bodies had no role in the design of the study and collection, analysis, and interpretation of data and in writing the manuscript.

\section{Availability of data and materials}

The datasets generated and/or analysed during the current study are not publicly available due to ethical and legal reasons, but are available from the corresponding author on reasonable request.

\section{Declarations}

Ethics approval and consent to participate

The patient was verbally informed of the nature and purpose of the report, and signed the informed consent forms.

\section{Consent for publication}

The patient provided signed consent for publication of the case.

\section{Competing interests}

The authors declare that they have no known competing financial interests or personal relationships that could have appeared to influence the work reported in this paper.

\section{Author details}

'Infectious Disease Department, No. 4th People's Hospital of Nanning and the Affiliated Nanning Infectious Diseases Hospital of Guangxi Medical University, Guangxi Medical University, No. 1, Second Lane, Changgang Road, Nanning 530023, Guangxi, China. ${ }^{2}$ Guangxi Key Laboratory of AIDS Prevention and Treatment \& Guangxi Universities Key Laboratory of Prevention and Control of Highly Prevalent Disease, School of Public Health, Guangxi Medical University, No. 22 Shuangyong Road, Nanning 530021, Guangxi, China. ${ }^{3}$ Guangxi Collaborative Innovation Center for Biomedicine, Life Sciences Institute, Guangxi Medical University, No. 22 Shuangyong Road, 530021 Nanning, Guangxi, China. ${ }^{4}$ Nursing College, Guangxi Medical University, No. 8 Shuangyong Road, Nanning 530021, Guangxi, China.

Received: 4 April 2021 Accepted: 24 August 2021

Published online: 16 September 2021

\section{References}

1. McCrary ML, Severson J, Tyring SK. Varicella zoster virus. J Am Acad Dermatol. 1999:41:1-14. quiz 5-6.

2. Low A, Gavriilidis G, Larke N, MR BL, Drouin O, Stover J, et al. Incidence of opportunistic infections and the impact of antiretroviral therapy among HIV-infected adults in low- and middle-income countries: a systematic review and meta-analysis. Clin Infect Dis. 2016;62:1595-603.

3. Gnann JW Jr. Varicella-zoster virus: atypical presentations and unusual complications. J Infect Dis. 2002;186(Suppl 1):91-8

4. Johnson RW, Levin MJ. Herpes zoster and its prevention by vaccination. Interdiscip Top Gerontol Geriatr. 2020;43:131-45.

5. Kennedy PG. Issues in the treatment of neurological conditions caused by reactivation of varicella zoster virus (VZV). Neurotherapeutics. 2016;13:509-13.

6. Gershon AA, Breuer J, Cohen Jl, Cohrs RJ, Gershon MD, Gilden D, et al. Varicella zoster virus infection. Nat Rev Dis Primers. 2015;1:15016.

7. Gilden D. Varicella-zoster virus infections. Continuum. 2015:21:1692-703.

8. Kedar S, Jayagopal LN, Berger JR. Neurological and ophthalmological manifestations of varicella zoster virus. J Neuro Ophthalmol. 2019;39:220-31.

9. Blank LJ, Polydefkis MJ, Moore RD, Gebo KA. Herpes zoster among persons living with HIV in the current antiretroviral therapy era. J Acquir Immune Defic Syndr. 2012;61:203-7.

10. Birlea M, Arendt G, Orhan E, Schmid DS, Bellini WJ, Schmidt C, et al. Subclinical reactivation of varicella zoster virus in all stages of HIV infection. J Neurol Sci. 2011;304:22-4.

11. Ansari R, Rosen LB, Lisco A, Gilden D, Holland SM, Zerbe CS, et al. Primary and acquired immunodeficiencies associated with severe varicella-zoster infections. Clin Infect Dis. 2020. https://doi.org/10.1093/cid/ciaa1274.

12. Domingo $\mathrm{P}$, Torres $\mathrm{OH}$, Ris J, Vazquez $\mathrm{G}$. Herpes zoster as an immune reconstitution disease after initiation of combination antiretroviral therapy in patients with human immunodeficiency virus type-1 infection. Am J Med. 2001;110:605-9.

13. Nagel MA, Niemeyer CS, Bubak AN. Central nervous system infections produced by varicella zoster virus. Curr Opin Infect Dis. 2020;33:273-8.

\section{Publisher's Note}

Springer Nature remains neutral with regard to jurisdictional claims in published maps and institutional affiliations.

Ready to submit your research? Choose BMC and benefit from

- fast, convenient online submission

- thorough peer review by experienced researchers in your field

- rapid publication on acceptance

- support for research data, including large and complex data types

- gold Open Access which fosters wider collaboration and increased citations

- maximum visibility for your research: over 100M website views per year

At BMC, research is always in progress.

Learn more biomedcentral.com/submissions 